\title{
CONTINUOUS PHASE MEASUREMENT IN THE W7-X INFRARED INTERFEROMETER BY MEANS OF A FPGA AND HIGH-SPEED ADCs
}

\author{
L. ESTEBAN, ${ }^{a *}$ M. SÁNCHEZ, ${ }^{a}$ J. SÁNCHEZ, ${ }^{a}$ P. KORNEJEW, ${ }^{b}$ M. HIRSCH, ${ }^{b}$ J. A. LÓPEZ, \\ A. FERNÁNDEZ, ${ }^{c}$ and $O$. NIETO-TALADRIZ ${ }^{\circ}$ \\ 'Laboratorio Nacional de Fusión, EURATOM-CIEMAT Association, Av'd Complutense N22, 28040 Madrid. Spain \\ 'Max-Planck-Institut fiir Plasmaphysik, EURATOM-IPP Association, Greifswald, Germany \\ ${ }^{\mathrm{c}}$ Departamento de Ingeniería Electrónica, ETSI Telecomunicación, Universidad Politécnica de Madrid, Madrid, Spain
}

Interferometry is used for measuring line average electronic densities in fusion plasmas. The W7-X stellarator will employ a two-color $\mathrm{CO}_{2}(10.591 \mu \mathrm{m})$ and $\mathrm{CO}$ $(5.295 \mu \mathrm{m})$ heterodyne-infrared interferometer as an electronic density measurement diagnostic. The frequency displacement is $40 \mathrm{MHz}$ for the $\mathrm{CO}_{2}$ wavelength and $25 \mathrm{MHz}$ for the $\mathrm{CO}$, so these values will fix the heterodyne frequencies. Because the frequency gap between the two carriers is wide enough and the detector sensitivity is similar for both wavelengths, it is possible to use a single detector for the two signals; nevertheless, they should be split with filters. Traditionally, the intermediate-frequency signals should be filtered, downconverted to a lower fre-

\section{INTRODUCTION}

An ordinary wave traveling through a plasma suffers a phase shift that under the assumption that the electronic density is much lower than the cutoff density is given by

$$
\phi=\phi_{p}+\frac{2 \pi \Delta l}{\lambda}
$$

The first term in Eq. (1) is the phase shift introduced by the electronic density variation, and the second term is due to mechanical vibrations and thermal drifts..$^{1,2}$ In our case of interest (infrared region), the phase shift introduced by mechanical vibrations is much higher than that caused by plasma fluctuations. This reason forces the use of a two-color or two-wavelength interferometer, where

\footnotetext{
*E-mail: luis.esteban-hemandez@ciemat.es
}

quency by the use of analog circuitry, and then processed. A new approach is proposed. The intermediatefrequency signals are directly sampled by means of high-speed analog-to-digital converters followed by a digital diplexer and a specific phase-meter processor implemented in a field-programmable gate array. Preliminary results from the $W 7-X$ infrared interferometer prototype, without plasma, are presented.

KEYWORDS: infrared interferometry, real-time processing. digital signal processing

Note: Some figures in this paper are in color only in the electronic version.

the second wavelength is used to cancel this undesirable contribution to the phase shift. In this case the final line average electronic density is calculated as ${ }^{1,2}$

$$
\int n_{e} d l=\frac{\Delta \phi_{1} \lambda_{1}-\Delta \phi_{2} \lambda_{2}}{p \cdot r_{e}\left(\lambda_{1}^{2}-\lambda_{2}^{2}\right)},
$$

where

$$
\begin{aligned}
& p=\text { integer that corresponds to the number of trips } \\
& \text { that the beams cross the plasma } \\
& r_{e}=\text { classical electron radius. }
\end{aligned}
$$

At W 7-X a single-channel two-color interferometer is under development dedicated to density control and cross-calibration of the Thomson scattering system. ${ }^{3}$ In addition, a multichannel interferometer (ten channels) ${ }^{4.5}$ is in preparation that presumably will use second-harmonic 
dispersion interferometry techniques as they are currently studied in detail at the TEXTOR tokamak. The single-channel system will use $\mathrm{CO}_{2}(10.591 \mu \mathrm{m})$ and $\mathrm{CO}$ $(5.295 \mu \mathrm{m})$ as the short wavelength, in a Mach-Zehnder configuration. In this type of arrangement, two probing beams cross the plasma, and two beams that travel the same optical distance outside the plasma are used as references; see Fig. 1. In the particular cases of using $\mathrm{CO}_{2}$ and $\mathrm{CO}$, the two interference signals can be detected using one single square law detector, provided that they can be separated afterward, with a considerable reduction in the number of optical components. In this contribution a diplexion scheme based on finite impulse response (FIR) digital filters is proposed, proving the viability of this approach and its advantages over an analog solution.

Comparing the phases of the output signals with two reference signals, line-integrated density can be recovered from Eq. (2). It is indispensable then to demodulate the phase information. Several basic steps are involved in this procedure. First, the signals should be preprocessed to meet the requirements in terms of number of samples per period and signal-to-noise ratio of the phasemeasuring algorithm; in the case of W7-X, the interference signals could be diplexed at this point. In a second stage the phase measurements should be computed using a proper algorithm, and finally, once the phase differences are obtained, the line-integrated density can be calculated with Eq. (2). This procedure is typically done with mixed-signal technology. In the TJ-II stellarator infrared interferometer, a novel phase-measuring system based in field-programmable gate arrays (FPGAs) and high-speed analog-to-digital converters (ADCs) is used, ${ }^{6}$ performing all the signal processing digitally, improving the bandwidth and the signal-to-noise ratio. In this contribution it is proposed to use the same approach used in the TJ-II infrared interferometer for the future W7-X infrared interferometer including several modifications. These modifications are described, and the first results obtained in the W7-X infrared interferometer test bench are discussed. This new processing concept will allow this diagnostic to provide a real-time signal for W7-X control systems. In the case of TJ-II, a general-purpose input/output parallel port is used to extract the density signal from the FPGA. As the response times of the control systems are in the 1-ms range, the velocity needs of this signal are much lower.

\section{PREPROCESSING AND FILTERING STAGE}

First, the output interference signals coming from the detector and their correspondent references should be digitized. The intermediate frequencies $\left(f_{I F}\right)$ are $40 \mathrm{MHz}$ for the probing and reference signals of $\mathrm{CO}_{2}$ and $25 \mathrm{MHz}$ for the $\mathrm{CO}$ signals. According to the Nyquist theorem, the sampling frequency should be $>80 \mathrm{MHz}$. The ADCs used are AD6645 from analog devices with a maximum sampling rate of 105 megasamples per second (MSPS), fulfilling the requirements of the application. At this point all the signals are digitized, and the $40-$ and $25-\mathrm{MHz}$ probing signals are superposed at the output of the ADC. Two approaches could be used to separate both signals. One approach would be to use analog filters before the ADCs (Ref. 7), and the other approach would be to filter digitally the signals after the sampling process. In both cases special care has to be taken with the phase responses of the filters in two-color interferometers. The next step involves deducing the conditions under which the filters will be operating and the type of filters that should be used with these conditions.

The phase shifts can be expressed as a function of the frequency shifts and the group delay of the filters as follows:

$$
\Delta \phi_{1}=\frac{d \phi_{1}}{d f} \Delta f_{1}
$$

and

$$
\Delta \phi_{2}=\frac{d \phi_{2}}{d f} \Delta f_{2} .
$$

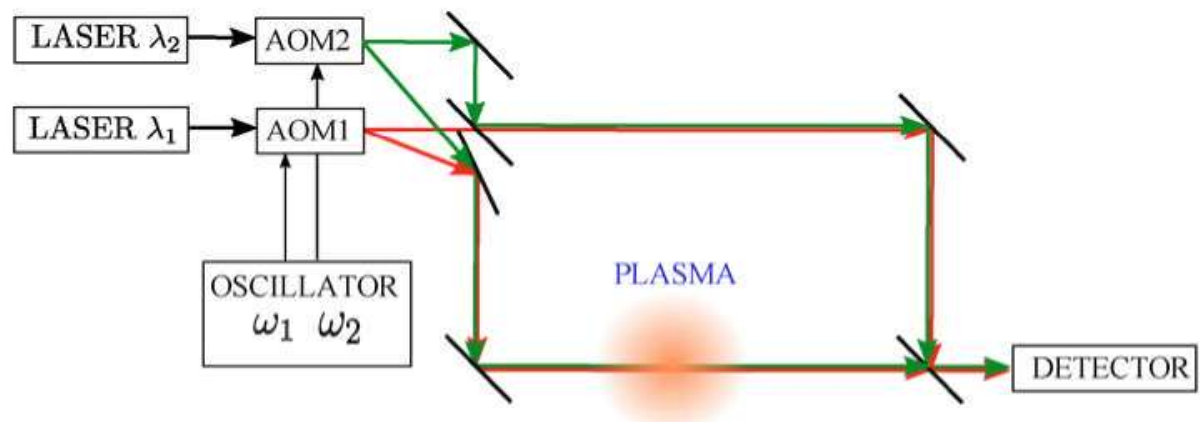

Fig. 1. Two-color one-channel heterodyne (infrared) interferometer scheme employing one single detector. The blocks are acoustooptic-modulators (AOMs), and they generate the heterodyne frequencies as well as the measuring and reference beams. 
From the optical path-length differences

$$
\lambda_{1} \Delta \phi_{1}=\lambda_{1} t \Delta f_{1}
$$

and

$$
\lambda_{2} \Delta \phi_{2}=\lambda_{2} t \Delta f_{2},
$$

one may state that $\Delta f_{1}$ and $\Delta f_{2}$ are related by a factor $m$ that is the relation between the two wavelengths as follows:

$$
m=\frac{\Delta f_{1}}{\Delta f_{2}}=\frac{\lambda_{2}}{\lambda_{1}} .
$$

In the absence of plasma the numerator of Eq. (2) should be equal to zero:

$$
\Delta \phi_{1} \lambda_{1}-\Delta \phi_{2} \lambda_{2}=0 .
$$

Substituting Eq. (3) and $\mathrm{Eq}$. (4) results in $\mathrm{Eq}$. (9):

$$
\frac{\Delta f_{1}}{\Delta f_{2}} \frac{d \phi_{1}}{d f}=\frac{\lambda_{2}}{\lambda_{1}} \frac{d \phi_{2}}{d f}=\frac{d \phi_{1}}{d f}=\frac{d \phi_{2}}{d f} .
$$

According to relation (9) the filters must have an equal group delay, or the slopes of their phase responses must be the same in order to correctly compute the lineintegrated density. These quantities are constant with linear phase filters. On the other hand, an equal group delay can be achieved using filters of the same order and bandwidth.

It is important to realize the difficulties in implementing analog filters ${ }^{7}$ with an exact phase linear response; this coupled with the lack of versatility of this approach and the processing capabilities of the FPGA have been the deciding factors as to electing a digital filtering approach.

Unlike other types of filters, FIR filters can be designed with an exact linear phase response, so the desired solution can be obtained easily. Two important parameters to take into account are the bandwidth and the attenuation in the rejection band. The optimal values of these parameters are calculated in a previous work. ${ }^{8}$ In these preliminary tests, two FIR bandpass digital filters have been designed with a bandwidth of $2 \mathrm{MHz}$ and an attenuation in the rejection band of $43 \mathrm{~dB}$ (to avoid cross talk), which results in filters of order 26 . The smoothing window chosen is a fixed Hann window, and the magnitude and phase responses of the filters can be seen in Fig. 2.

After separating both carriers, the numbers of samples per period are 2.625 for the $40-\mathrm{MHz}$ signals and 4.2 for the $25-\mathrm{MHz}$ signals, which are insufficient for the phase-measuring algorithm used ${ }^{12}$; this algorithm requires a minimum of at least four samples per period, and the measurement error decreases as this number increases.

To increase the number of samples per period, one approach is to change the sampling rate. This technique is usually used in digital signal processing ${ }^{9.10}$ and can be achieved via downsampling and upsampling blocks; see Fig. 3.

First, an M-fold decimation is done to all the signals, the sampling rate is reduced, and an alias of each signal is obtained. For the $40-\mathrm{MHz}$ signals the decimation is made by a factor of 3 ; the process is like sampling a signal of $(40-105 / 3) \mathrm{MHz}=5 \mathrm{MHz}$ with a sampling rate of 35 MSPS, having seven samples per period. For the $25-\mathrm{MHz}$ signals, the decimation factor is 4 , performed in a similar manner as before; this is equivalent to sampling a signal of $1.25 \mathrm{MHz}$ with a sampling rate of 25 MSPS, which results in 20 samples per period. So far, undersampled aliases have been obtained at different sampling rates. To continue with the phase measurement process, the sampling rates should be equalized for all the signals. We can recover the original sampling rate of 105 MSPS and continue increasing the number of samples per period using upsampling blocks (zero padding). The only drawback is that interpolation filters should be included in the output of the upsamplers, which introduces a small latency in the processing pipeline. The upsampling factors are 3 and 4 for the $40-$ and $25-\mathrm{MHz}$ signals, respectively. This process is summarized in Fig. 3, and in Fig. 4 the frequency displacement is illustrated.

The output signals to the preprocessing block spectrum can be seen in Fig. 5. The whole procedure corresponds to a digital frequency downconversion, and replicas of the 40 - and $25-\mathrm{MHz}$ signals are obtained at 5 and $1.25 \mathrm{MHz}$, respectively, with 21 and 80 samples per period, increasing the performance of the phasemeasurement process that will be explained in Sec. III.

This procedure was formerly performed with ana$\log$ frequency mixing stages that have been a proven source of errors due to intermodulation, electrical cross talk, and coupling of spurious signals. With the new concept presented in this contribution, there is no longer any need to use this type of preprocessing analog stages, improving the signal-to-noise-ratio of the sampled interference signals.

\section{PHASE DETECTION PROPOSAL FOR W7.X}

After conditioning the signals, the phase measurements can be performed. The algorithm used in the first trials is an algorithm that is used in the TJ-II infrared interferometer. ${ }^{11.12}$ In its first version this algorithm was tested in an off-line processing system, ${ }^{12}$ and more recently, it has been implemented in an FPGA-based phase meter with online processing capabilities. ${ }^{6}$ It is precisely this second version that has been tested in the infrared interferometer prototype of W7-X with the modifications described in Sec. II.

The phase measurement algorithm is summarized in Fig. 6 and is explained in detail in Refs. 11 and 12; its causal version is in Ref. 6. 


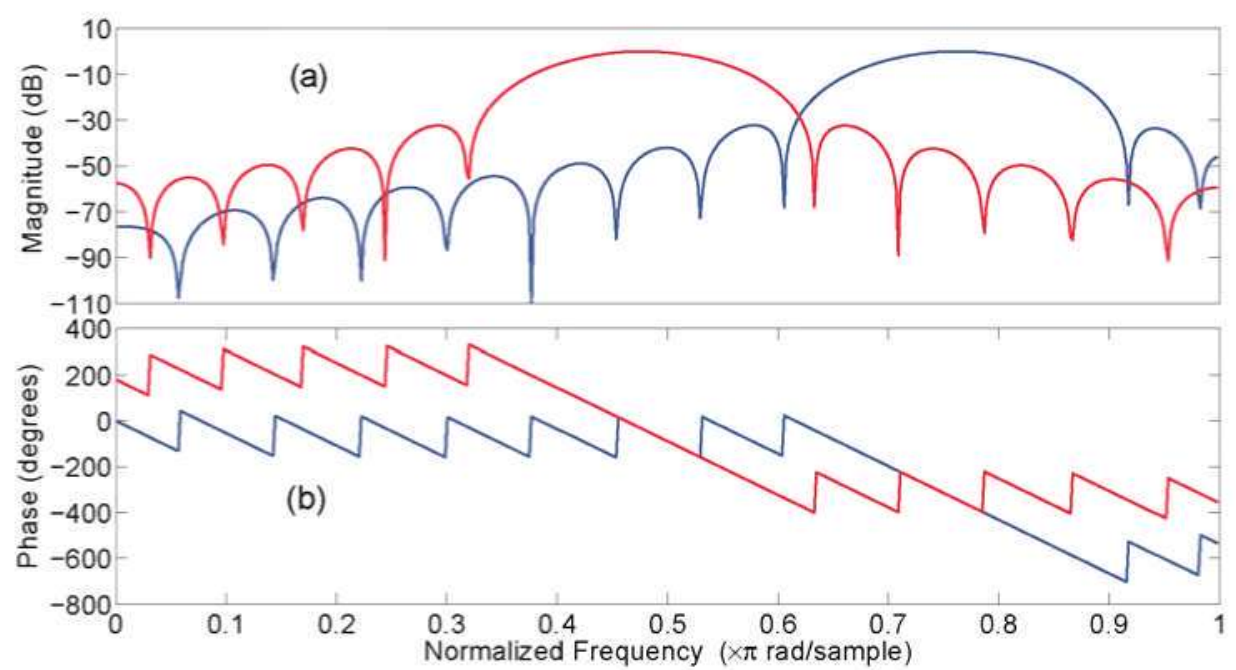

Fig. 2. Transfer function of the frequency diplexion filters. One filter has its low and high cut off frequencies located at $f_{c l}=$ $24 / 52.5$ and $f_{c h}=26 / 52.5$, and the other filter has its low and high cutoff frequencies located at $f_{c l}=39 / 52.5$ and $f a_{c h}=$ 41/52.5. (a) The detail of the magnitude response is shown. The red and blue lines correspond to the magnitude response of the 25- and $40-\mathrm{MHz}$ filters, respectively. An attenuation for each carrier $>43 \mathrm{~dB}$ is achieved. (b) The unwrapped linear phase response of the filters can be seen. The phase responses are shown. The red and blue lines correspond to the linear phase response of the $25-$ and $40-\mathrm{MHz}$ filters.

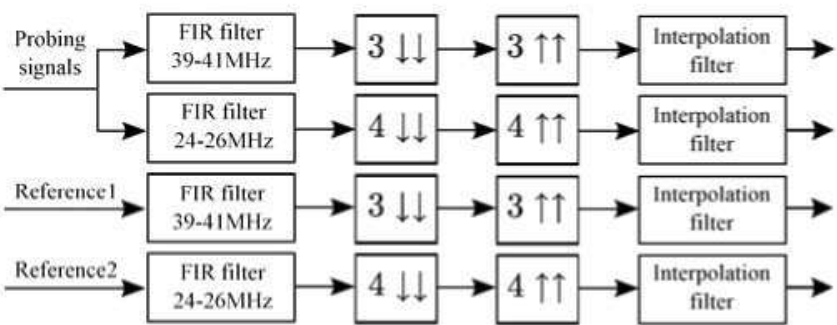

Fig. 3. Online processing block diagram. The down arrows indicate that the sampling rate is reduced by 3 and 4 for the $\mathrm{CO}_{2}$ and $\mathrm{CO}$ signals, respectively. The up arrows indicate an increase in the sampling rate of 3 and 4 in a similar manner. In this case zeros are introduced between the samples. With this procedure aliases of the original signals are obtained but with more samples per period than the original signals (digital frequency downconversion).

This phase measurement algorithm is based on linear interpolation around the zero. And, the phase is computed for every sample time $i$ as

$$
\phi_{i}=2 \pi n+\frac{2 \pi\left[(i-j)-\tau_{n}\right]}{t_{n+1}-t_{n}},
$$

where

$$
\begin{aligned}
\tau_{n} & =\frac{\left|S_{j}\right|}{\left|S_{j}\right|+\left|S_{j+1}\right|} \Delta t \\
t_{n} & =i \Delta t+\tau_{n}
\end{aligned}
$$

$$
\begin{aligned}
\tau_{n}= & \text { time between the latter sample and the } \\
& \text { next zero cross } \\
\left|S_{j}\right|,\left|S_{j+1}\right|= & \text { signal amplitudes before and after zero } \\
& \text { cross } j \\
t_{n}= & \text { signal period correspondent to the } n \\
& \text { zero cross; see Fig. } 6 .
\end{aligned}
$$

An important issue in phase measurements for interferometers is that the zero crossings $j$, Fig. 6 , should be counted because the total phase shifts of the signals are typically higher than several periods. A miscounting of a zero cross (fringe jump) results in a cumulative error of $2 \pi$ per fringe missed. There are two causes of these phenomena:

cause 1: fringe jumps because of uncertainty in the direction of the rotation of the phase and hence of the frequency

cause 2: a low enough signal-to-noise ratio in the input signals. This can be caused by vanishing of the signals because of edgelocalized modes (ELMs), pellet injection, and other plasma instabilities.

Cause 1 is related to the phase measurement procedure and should be taken into account when making a frequency downconversion. It can be ensured that the direction of rotation is positive and the uncertainty can be eliminated if the condition $1 /(2 \pi d \phi) / d t<f_{I F}$ is met. In an ideal case, $f_{I F}=B W / 2$, where $B W$ is the 


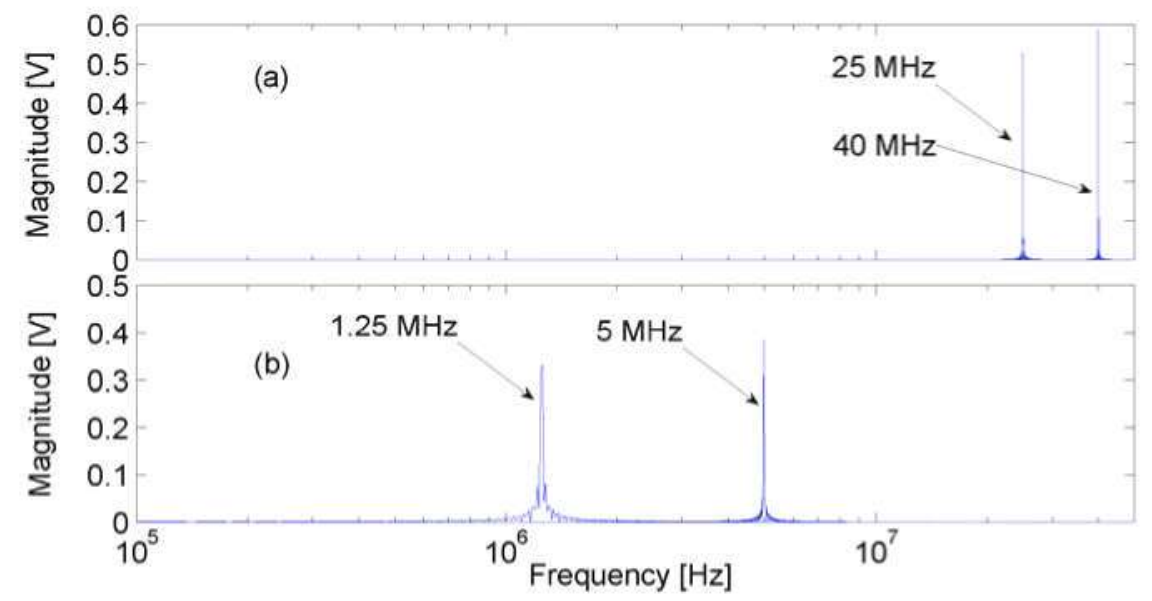

Fig. 4. Spectra shown in (a) would correspond to the two output signals ( 25 and $40 \mathrm{MHz}$ ) of the diplexor. In the preprocessing stage these signals are downconverted to 1.25 and $5 \mathrm{MHz}$, preserving the original $105 \mathrm{MSPS}$ sampling rate.

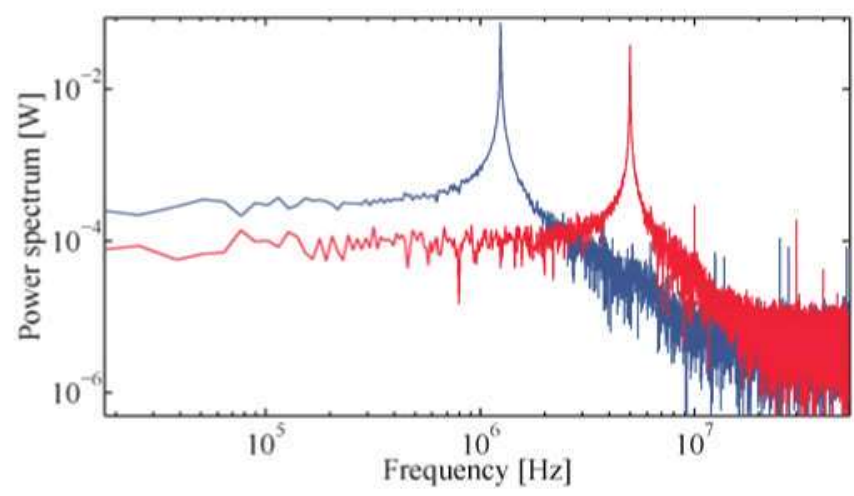

Fig. 5. Real diplexed signals of the W7-X infrared interferometer prototype. The blue and red lines correspond to the $\mathrm{CO}$ and the $\mathrm{CO}_{2}$ frequency downconverted interference signals, respectively. It can be seen that the frequency of these signals is 1.25 and $5 \mathrm{MHz}$.

bandwidth of the signal over the carrier $f_{I F}$. In the case of interest, the maximum $B W$ would be $2 \times 1.25 \mathrm{MHz}=$ $2.5 \mathrm{MHz}$ correspondent to the $f_{I F}$ after performing the digital frequency downconversion to the $\mathrm{CO}$ signals. The second reason is more related to the physical characteristics of the diagnostic itself. Critical plasma densities in interferometers that operate at millimeterto-submillimeter wavelengths such as the JET farinfrared interferometer ${ }^{13}$ are lower than that of infrared interferometers. With a low critical cut of densities, the phenomena explained in cause 2 can cause a temporary reflexion or deflexion of the probing beams with the consequent reduction in the detected amplitudes or even their disappearance. For these reasons far-infrared interferometers are more sensitive to changes in the plasma density caused for example by the phenomena explained in cause 2 .

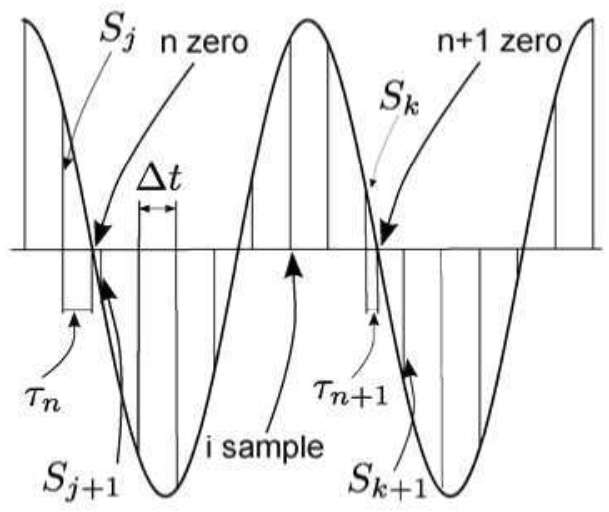

Fig. 6. Phase-measuring interpolation algorithm.

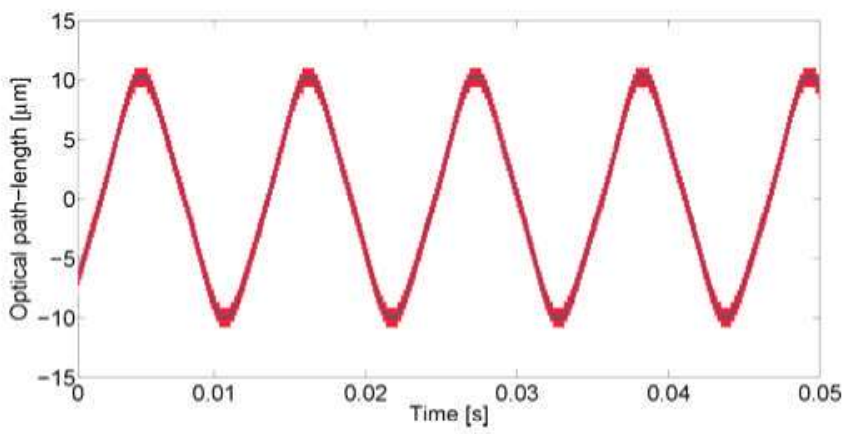

Fig. 7. Vibration test performed with a piezoelectric oscillator moving one of the mirrors of the interferometer. The optical path variations are illustrated at the maximum output sampling rate: 105 MSPS. The red and blue lines correspond to the optical path-length differences of the $\mathrm{CO}_{2}$ and $\mathrm{CO}$, respectively. The variations of the optical paths are in agreement with the $90-\mathrm{Hz}$ sinusoidal function introduced with the piezoelectric oscillator. 
The above phase measurement algorithm has been tested for several campaigns for the TJ-II infrared interferometer with no fringe jumps in the computation of the integrated electron density, so it is a good choice for the future W7-X infrared interferometer. Perhaps other algorithms can be tested using the same FPGA onlineprocessing concept.

\section{RESULTS}

Once the signal-processing blocks including the phase measurement algorithm used are defined, the next step is to test the system. For this purpose in the arrangement of Fig. 1, a vibrating mirror has been included in the measuring paths, modulating the phase of the interferometer. Denoting by $\Omega$ the vibrating frequency of the mirror, from the second term of Eq. (1), one may write $\Omega=2 \pi \Delta l / \lambda_{\mathrm{CO}_{2}}$ and $\Omega=2 \pi \Delta l / \lambda_{\mathrm{co}}$. These frequencies should be detected when demodulating the phase of the interference signals individually. Figure 7 gives the results of this demodulation with a vibrating frequency of $\Omega=90 \mathrm{~Hz}$ and at an equivalent sampling rate of 105 MSPS. The noisy line and the red line correspond to the $\mathrm{CO}_{2}$ and $\mathrm{CO}$ optical path lengths, respectively. The $\mathrm{CO}_{2}$ optical path is much more noisy than that of the $\mathrm{CO}$ because the number of samples per period in the $\mathrm{CO}$ reference and probing signals is much larger than those of the $\mathrm{CO}_{2}: 80$ versus 21 , respectively.

To limit the noise, a simple averaging process is performed: reducing the sampling rate in the final optical path-length compensation, which is calculated subtracting both averaged paths, the numerator of $\mathrm{Eq}$. (2). Figure 8 shows the compensation of the optical path length for an equivalent sampling rate of 10.5 kilosamples per second (kSPS). Figure 9 illustrates the spectrum of the compensated signal, where the very small peak located at $90 \mathrm{~Hz}$ is because the paths are not totally compensated. Among this peak the standard deviation of the compensated signal is $\sim 0.01 \mu \mathrm{m}$, being the resolution expressed in terins of electron average density of $\Delta(n d l) \simeq 0.1 \times$

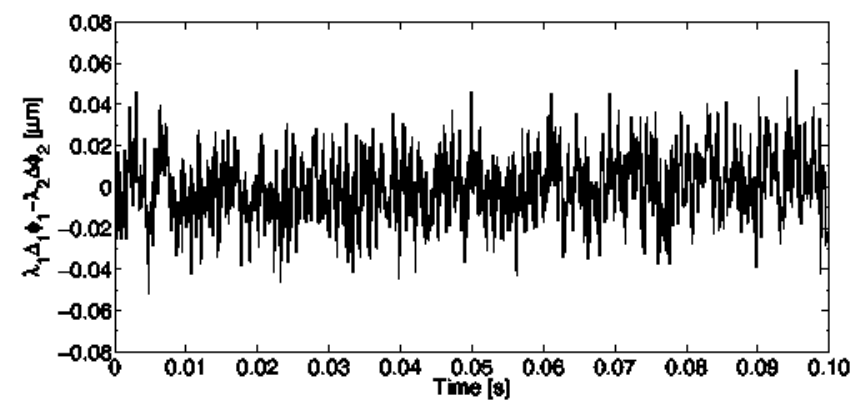

Fig. 8. Compensation of the optical path as the result of subtracting the optical paths measured by both interferometers.

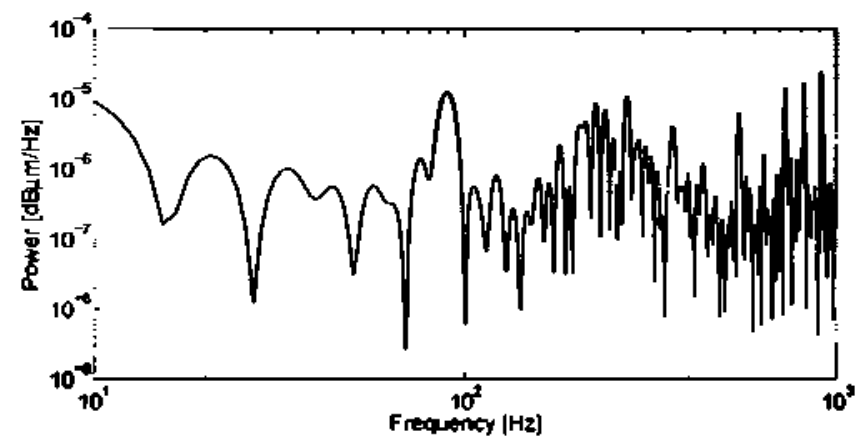

Fig. 9. Spectrum of the compensated optical path. The peak at $90 \mathrm{~Hz}$ corresponds to a misalignment of the interferoneter; however, the accuracy obtained is very good.

$10^{18} \mathrm{~m}^{-2}$. With these new values the required precision to derive a correct line-integrated density measurement could be obtained without averaging or filtering.

Figure 10 plots the relation between resolution in terins of standard deviation (error) and sampling rate. Some promising results can be seen. For example, for an output sampling rate of $2 \mathrm{kSPS}$, the resolution obtained in the measurement is $-10^{-2} \mu \mathrm{m}$. These measurements could be improved by changing the intermediate frequency of the $\mathrm{CO}_{2}$ to a value of $-36.25 \mathrm{MHz}$ instead of $40 \mathrm{MHz}$, so the number of samples per period after performing the digital downconversion would be 80 instead of 21 for $\mathrm{CO}$.

\section{CONCLUSIONS}

A new online processing concept based on FPGAs and a novel phase-ineasuring algorithm have been tested successfully in the W7-X infrared interferometer prototype, and promising results have been obtained. The one-detector arrangement illustrated in Fig. 1 has been proven to be feasible, demonstrating several benefits over the two-detector scheme, which includes reducing the optical cross talk and the number of optical components used; nevertheless, an electronic diplexer is needed to separate both carriers. This diplexer has been implemented digitally in the FPGA showing its advantages over the analog filtering solution, ${ }^{7}$ especially in terms of versatility and resolution versus bandwidh response. Also, a sampling rate alteration stage has been included in the design to increase the number of samples per period of the signals, hence improving the quality of the final pathlength compensation.

Resolutions of $10^{-2} \mu \mathrm{m}$ at an output rate of $10 \mathrm{kSPS}$ have been achieved in the preliminary tests. The system should be modified so as to be capable of measuring during long periods ( $\simeq 30 \mathrm{~min}$ ), which is indispensable for W7-X operation needs. Several modifications can be 


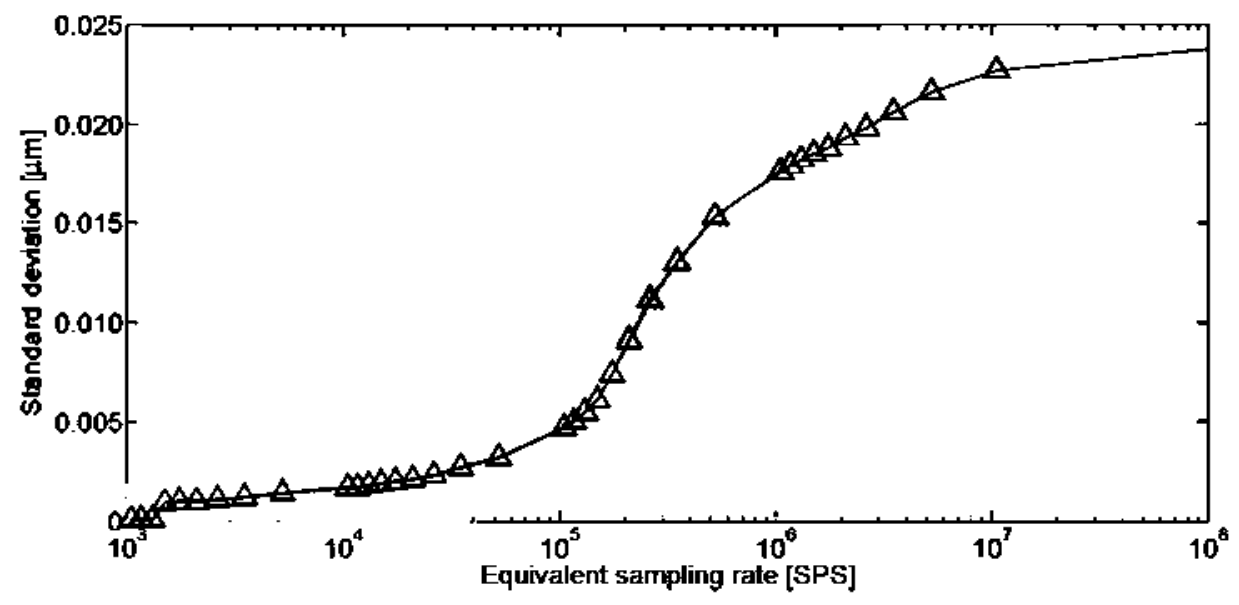

Fig. 10. Accuracy obtained for several sampling rates. For the detection of quick changes, the resolution could worsen, and the temporal resolution increases. As can be seen for an equivalent sampling rate of $105 \mathrm{kSPS}$, there is much more resolution than needed for the former purpose: $1 / 2000$ of a $\mathrm{CO}_{2}$ fringe. Typically, ELMs and other quick changes occur of the order of $40 \mathrm{kHz}$. In this case the resolution limit is not of a random nature but is deterministic, so the performance can be further improved with better alignment.

made to this design in order to improve the results. First, a compromise between the intermediate frequencies and the number of samples per period should be established; for instance, an intermediate frequency of $36.25 \mathrm{MHz}$ for the $\mathrm{CO}_{2}$ would result in 80 samples per period, and the conespondent resolution would be inuch better. Another important issue is that the causal version of the interpolation algorithm ${ }^{6}$ uses the former period to calculate the actual period phase parameters, which causes an error that increases as the phase acceleration increases. This can be easily solved by introducing a latency in the pipeline of the phase-measuring processor.

\section{REFERENCES}

1. H. J. HARTFUSS et al., Plasma Phys. Control. Fusion, 39, 1693 (1997).

2. I. H. HUTCHINSON, Principles of Plasma Diagnostics, Cambridge University Press (2008).

3. P. KORNEJEW et al., Rev: Sci. Instrum., 77, 10F323-1 (2006).
4. H. DREIER et al., Rev. Sci. Instrum., 79, 10E712 (2008)

5. H. DREIER et al., "Performance Analysis for an Infrared Second Harnonics Dispersion Interferometer," presented at 36th Int. Cont. Plasma Science and 23rd Symp. Fusion Engineering, San Diego, California.

6. L. ESTEBAN et al., Fusion Eng. Des. (2010) doi:10.1016/ j.fusengdes.2010.03.036.

7. CANTON et al., Appl. Optics, 45, 36 (2006).

8. M. SANCHEZ et al., AIP Conf. Proc., 993, 187 (2008).

9. S. K. MITRA, Digital Signal Processing, McGraw-Hill (2006).

10. B. FARHANG-BOROUJENY, Signal Processing Techniques for Software Radios, Lulu (2008).

11. P. V. ZUBAREV et al., Instrum. Exp. Technol, 46, 2, 171 (2003)

12. M. SANCHEZ et al., Rev. Sci. Instrum., 75, 10 (2004).

13. L. ZABEO et al., Rev. Sci. Instruni, 74, 3645 (2003). 